\title{
Energy dissipation of the liquid in systems with a hydrophobic surface
}

\author{
Simona Fialová, František Pochylý, Martin Hudec
}

Faculty of mechanical engineering, Brno University of Technology, Technicka 2896/0, Kralovo Pole, 61669, Brno, Czech Republic

\begin{abstract}
The work is divided into theoretical and experimental parts. In the theoretical part the viscous forces performance for both laminar as well as turbulent flow of compressible fluids are defined. It is split into the viscous forces useful power and the power corresponding to mechanical losses. This part is expressed by the dissipation function. It is shown that the dissipation function considering hydrophobic surfaces cannot be determined from the pressure difference. It is necessary to take into account the slip of the liquid over the hydrophobic surface which depends on the value of surface energy. The values of surface energy for different types of hydrophobic surfaces, obtained by experiment, are shown within this work. The new definition of the dissipation function for hydrophobic surfaces is presented depending on the adhesion coefficient, also determined by an experiment. The theoretical part of the work is complemented by experiment. The experiment is aimed at the differential pressure measurement in a circular cross section pipe. The results are processed for both laminar and turbulent stationary flow in relation to the flow. Both hydrophilic and hydrophobic surfaces and their mutual correlation are taken into consideration. On the basis of the new mathematical model for hydrophobic surfaces the value of dissipation function is determined depending on the slip velocity of the liquid along the hydrophobic surface.
\end{abstract}

\section{Introduction}

Hydrophobicity 8 is related to the effect of adhesion forces between the surface and the liquid. The degree of hydrophobicity is assessed on the basis of the adhesion of the droplet to the surface, represented by the contact angle $\theta 1,2$. Angles $\theta>80^{\circ}$ characterize hydrophobic surfaces, angles $\theta>120^{\circ}$ characterize so called superhydrophobic surfaces and surfaces associated with angles $\theta>150^{\circ}$ are ultrahydrophobic. This characteristic of the surface to repel liquid was assumed by Navier 7, 200 years ago. For liquid on hydrophobic surface, he predicted the shear stress to be proportional to the slipping velocity. Within the experimental part of the woldwide work the description of the behavior of liquids in the vicinity of hydrophobic walls affected only 13 , who processes the analytical solutions of the motion of a group of continuous variable systems - everything is simplified to the plane; and 13 who shows two types of determination of the motion tensor that characterizes straight hydrophobic surface for any slip length. It is clear that the experimental determination of the influence of hydrophobicity is clearly easier than its analytical description, which would lead to the possibility of using for simulations in commercial softwares. Experiments describing the flow behavior after hydrophobic and superhydrophobic surfaces are covered by series of authors. E.g. 10 who studied the wettability effect on the stability of flow and transition to turbulent flow. The slip boundary condition on the wall is given as a velocity proportional to the velocity gradient and slip length. Calculations on the wall used linearized Navier-Stokes equations for 2D flow (OrrSommerfeld equations). Their conclusion is that the transition to turbulence is slowed in the direction of the streamline and accelerated in the direction perpendicular to it.

Expected reduction in losses due to friction decrease on the wall by hydrophobic and ultrahydrophobic surfaces are experimentally verified eg. in 13 where the authors studied the ability of superhydrophobic 
materials to reduce friction losses in laminar and turbulent flow regimes. They predict the emergence of the air layer on the superhydrophobic surface. They used PIV and pressure measurement in the droplets. They also use the Navier model [7] of the slippage (again velocity gradient and length of slip).

The fact that the superhydrophobic materials have the potential to reduce friction by forming an air layer (plastron) on the surface refers e.g. 12. Numerical simulation is performed using the two-phase laminar flow. Using Navier [7] slip model (velocity gradient and length of slip) they point out that the Navier model simulates only the mean value but does not simulate the interaction between the surface and the air and between the surface portions and the outer liquid stream. The results show losses reduction in Stokes flow by $19 \%$ in the water-air system. When increasing the Reynolds number to 100 , the losses will reduce by $50 \%$ (assuming a viscous friction and suppressing the plastron separation). Constant velocity slippage and losses reduction due to plastron cause a complete suppression of the turbulent flow when flow is torn off, but is still stable $(24<\operatorname{Re}<130)$. Important is the recognition that helps to the losses reduction is more the detailed surface geometry than averaged influence, which is expected to reduce overall losses.

Reduction of losses of $40 \%$ and the length of the slip to 20 microns for ultrahydrophobic surface states as a result of a series of experiments 9. The losses reduction by using a hydrophobic surface has not been proven.

The importance of hydrophobic surfaces for the flow in tube of $16 \mathrm{~mm}$ diameter, where occurred under laminar flow the losses reduction of $14 \%$ is demonstrated in 5. Mathematically, again using the Navier-Stokes equations, where is introduced the slip coefficient. The same coefficient is implemented in the loss coefficient $\lambda$. They conducted experiments to verify the theory in small diameter tubes (6 and 12 $\mathrm{mm}$ ) and the coating to about 10 microns.

In fact all the known research of the viscous liquid flow over hydrophobic surfaces was made in small diameters tubes and low Reynolds numbers (including the uncerntainty of the Reynolds number value for the flow with assumed air plastron) The degree of hydrophobicity determines the magnitude of slippage velocity of the liquid over the surface 3. Slip conditions vary for laminar and turbulent flow 3. In case of laminar flow a thin layer of air between the surface and the liquid is formed as an effect of adhesion and intermolecular forces. The thickness of the layer depends on the Reynolds number. At higher Reynolds numbers, the layer gets thinner until it dissappears. This corresponds to the size of the slip velocity on the wall. When the air layer disappeares, the liquid is slipping, provided that the shear stress $\tau$ exceeds a certain critical value $\tau_{k}$. The slippage exists, if it holds:

$|\tau|>\left|\tau_{k}\right|$.

The slip of the liquid is in this case governed by the new boundary condition 1 . For the flow in the pipe of constant cross-section the following applies:

$\tau=-k v$.

Another situation may occur when a turbulent flow develops. Given that the velocity on the hydrophobic surface is not zero, there will be nonzero values of turbulent viscosity in contrast to hydrophilic surfaces whereupon the liquid adheres to. And this fact can significantly affect energy dissipation during the flow of a liquid over a hydrophilic/hydrophobic surface.

\section{Energy dissipation}

The power consumption $P$ required to cover the hydraulic losses is determined by the dissipation function $\mathcal{D}$ in the form described in the 5 as Rayleigh friction:

$\mathcal{D}=\frac{1}{2} P=\frac{1}{2} \int_{V} \Pi_{i j} \frac{\partial v_{i}}{\partial x_{j}} d V$.

A turbulent flow is characterized by significant unsteady pulsating velocity components $v_{i}^{\prime}(\bar{x}, t)$, that can have both positive and negative values. It is assumed that the average value for a sufficiently long period of time $T$ is equal to zero. Thus:

$\frac{1}{T} \int_{t-\frac{T}{2}}^{t+\frac{T}{2}} v_{i}^{\prime}(\boldsymbol{x}, \tau) d \tau=0$.

When we decompose the resulting velocity $\boldsymbol{v}(\boldsymbol{x}, t)$ to its components:

$\boldsymbol{v}=\widehat{\boldsymbol{v}}(\boldsymbol{x}, t)+\boldsymbol{v}^{\prime}(x, t)$,

Than for $\widehat{\boldsymbol{v}}$, in regards to (2.2), holds the relation:

$\widehat{\boldsymbol{v}}=\frac{1}{T} \int_{t-\frac{T}{2}}^{t+\frac{T}{2}} \boldsymbol{v}(\boldsymbol{x}, \tau) d \tau$. 
For the average value of the dissipation function can be analogously written:

$2 \widehat{\mathcal{D}}=\widehat{\boldsymbol{P}}=$
$=\frac{1}{T} \int_{t-\frac{T}{2}}^{t+\frac{T}{2}} \int_{\boldsymbol{V}} \widehat{\Pi}_{i j}(\boldsymbol{x}, \tau) \frac{\partial \widehat{v}_{l}(\boldsymbol{x}, \tau)}{\partial x_{j}} d V d \tau$.

The term (2.5) can be in the case of steady flow corrected to:

$2 \widehat{\mathcal{D}}=\widehat{\boldsymbol{P}}=\int_{\boldsymbol{V}} \widehat{\Pi}_{i j}(\boldsymbol{x}) \frac{\partial v_{i}(\boldsymbol{x})}{\partial x_{j}} d V$.

$\widehat{\Pi}_{i j}=2\left(\eta+\eta_{T}\right) \hat{\mathrm{v}}_{i j}-\frac{2}{3} \rho k \delta_{i j}$

where $\eta$ - molekular viscosity, $\eta_{T}=\eta_{T}(\boldsymbol{x})$ is the turbulent viscosity, $k$ is the kinetic energy density of dissipation for the turbulence model $k-\varepsilon$. [4] It holds:

$k=\frac{1}{T} \int_{t-\frac{T}{2}}^{t+\frac{T}{2}} v_{i}^{\prime} v_{i}^{\prime} d \tau$.

$\eta_{T}=\rho C_{\mu} \frac{k^{2}}{\varepsilon} ; \quad C_{\mu}=$ konst.

$\varepsilon=v \frac{1}{T} \int_{t-\frac{T}{2}}^{t+\frac{T}{2}} \frac{\partial v_{i}^{\prime}}{\partial x_{j}} \frac{\partial v_{i}^{\prime}}{\partial x_{j}} d \tau, \quad v=\frac{\eta}{\rho}$.

From the definition of $(2.8) \div(2.10)$ result the following conclusions.

\section{Hydrophillic surface}

The condition of liquid adhesion holds for the hydrophillic surface. On the immobile wall then holds:

$\boldsymbol{v}=0 \Rightarrow \widehat{w}=0 ; \boldsymbol{v}^{\prime}=0 ;$

$k=0 ; \eta_{T}=0 ; \varepsilon \neq 0$.

\section{Hydrophobic surface}

Nonwettable wall is characterized by so called hydrophobic surface that repels the liquid which slides over the surface and thus the velocity is considered as non-zero. Contrary to the wettable wall, the following conditions can be written:

$\boldsymbol{v} \neq 0 ; \widehat{\boldsymbol{v}} \neq 0 ; \boldsymbol{v}^{\prime} \neq 0$;

$k \neq 0 ; \eta_{T} \neq 0 ; \varepsilon \neq 0$.
These conditions will significantly affect the value of the dissipation function.

The dissipation function can be set from the NavierStokes average equations, considering for example the $k=\varepsilon$ turbulence model. For the incompressible fluid they are expressed in the form:

$\rho \frac{\partial}{\partial x_{j}}\left(\hat{v}_{i}, \hat{v}_{j}\right)-\frac{\partial \widehat{\Pi}_{i j}}{\partial x_{j}}+\frac{\partial \hat{p}}{\partial x_{i}}=0$

$\hat{p}=\frac{1}{T} \int_{t-\frac{T}{2}}^{t+\frac{T}{2}} p(\boldsymbol{x}, \tau) d \tau=\hat{p}(\boldsymbol{x})$.

From the expression (2.13), the dissipation function can be derived by integration over the multiple contiguous area $V$ as:

$$
\begin{aligned}
2 \widehat{\mathcal{D}}= & \widehat{P}= \\
= & -\left(\frac{\rho}{2} \int_{S} \hat{v}_{m} \hat{v}^{2} d S+\int_{S} \hat{p} \hat{v}_{m} d S\right)+ \\
& +\int_{S} \widehat{\Pi}_{i j} m_{j} \hat{v}_{i} d S+\int_{\Gamma} \widehat{\Pi}_{i j} \hat{v}_{i} m_{j} d \Gamma \\
\int_{S} p m_{i} d S & =\int_{S} \widehat{\Pi}_{i j} m_{j} d S+ \\
& +\int_{\Gamma} \Pi_{i j} m_{j} d \Gamma-\rho \int_{S} \hat{v}_{i} \hat{v}_{m} d S
\end{aligned}
$$

Where $\hat{v}_{m}=\hat{v} \cdot \mathbf{m}, \mathbf{m}-$ the nominal vector of the outer normal to the liquid, $S$ - the input/output surface, $\Gamma$ - rigid surface (wall).

If the term (2.15) is simplified for the simply contiguous area formed by the pipe of constant crossection, it holds:

$$
\begin{aligned}
& 2 \widehat{\mathcal{D}}=\hat{P}=\int_{\Gamma} \widehat{\Pi}_{i j} \hat{v}_{i} m_{j} d \Gamma-\int_{S} \hat{p} \hat{v}_{m} d S \\
& \int_{S} p m_{i} d S=\int_{\Gamma} \Pi_{i j} m_{j} d \Gamma
\end{aligned}
$$

For a cirrcular cross section of the pipe, (2.18) can be written in the form: 
$\Delta \hat{p}=\frac{\Gamma}{S}\left(\eta+\eta_{T}\right)|\operatorname{tg} \alpha|$,

where $S=\pi R^{2} ; \Gamma=2 \pi R L, \quad R$ - pipe radius, $\alpha-$ velocity profile angle on surface $\Gamma,|\operatorname{tg} \alpha|=\frac{d v_{T}}{d r}, L-$ pipe length.

Considering that in the given case it holds $v_{T}=$ $\left(v_{1}, 0,0\right)$, the term $(2.17)$ can be modified to the form:

$$
2 \widehat{\mathcal{D}}=\widehat{\mathrm{P}}-v_{T} \Gamma\left(\eta+\eta_{T}\right)|\operatorname{tg} \alpha|+\Delta \hat{p} v_{S} S
$$

After substitution in equation (2.19) we obtain a very important result:

$$
2 \widehat{\mathcal{D}}=\widehat{\mathrm{P}}=\left(\eta+\eta_{T}\right) \Gamma\left(v_{S}-v_{\Gamma}\right)|\operatorname{tg} \alpha|
$$

It is obvious from terms (2.19), 2.20) that the turbulent viscosity value and $|\operatorname{tg} \alpha|$ will significantly affect the dissipation function.

\section{The dependence of the dissipation function on the velocity profile shape}

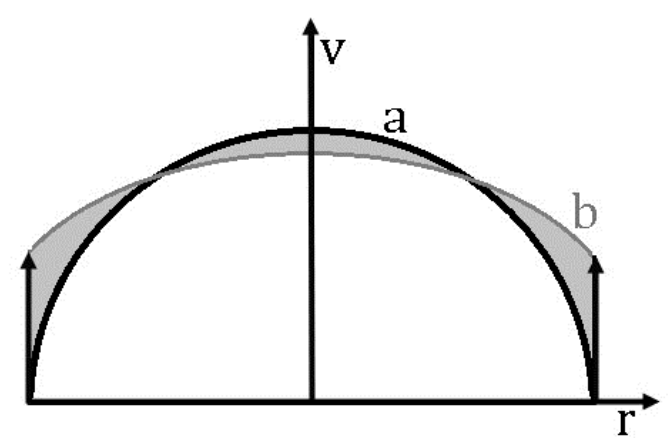

Fig. 1 Velocity profile shape a - hydrophilic, b - hydrophobic surface

When laminar flow is considered, terms (2.18), (2.20) can be written in the form:

$\Delta p=\frac{\Gamma}{S} \eta|\operatorname{tg} \alpha|$

$2 \mathcal{D}=\eta \Gamma\left(v_{S}-v_{\Gamma}\right)|\operatorname{tg} \alpha|$.

From Fig. 1, it is obvious that:

$\left|\operatorname{tg} \alpha_{a}\right|>\operatorname{tg} \alpha_{b}$

Given that for laminar flow $\eta=$ const. it is clear that slippage of the liquid will reduce the pressure difference $\Delta p$ as well as the hydraulic losses and the value of the dissipation function. This is valid for the case of slippage due to an air layer, as well as when it disappears.
Another situation occurs for the turbulent flow, when both the pressure difference and the dissipation function will be affected by nonzero turbulent viscosity.

In that case, it holds:

$\Delta \hat{p}=\frac{\Gamma}{S}\left(\eta+\eta_{T}\right)|\operatorname{tg} \alpha|$

$2 \widehat{\mathcal{D}}=\widehat{\mathrm{P}}=\left(\eta+\eta_{T}\right)|\operatorname{tg} \alpha|\left(v_{S}-v_{\Gamma}\right)$

From here it is visible that if $\left|\operatorname{tg} \alpha_{b}\right|<\left|\operatorname{tg} \alpha_{a}\right|$ then probably $\eta_{T}>\eta$. Therefore despite of slippage on the wall, it can not occur that both the differential pressure and dissipation function will grow compared to the case of laminar flow.

For example:

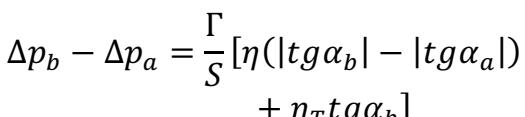

$$
\begin{aligned}
& \Delta p_{b}-\Delta p_{a}=\left[\left(\eta+\eta_{T}\right) \operatorname{tg} \alpha_{b}-\eta \operatorname{tg} \alpha_{a}\right]
\end{aligned}
$$

\section{Boundary condition on the hydrophobic surface}

In the above mentioned hypotheses we have focused only on the fluid slip along the hydrophobic surface. For these considerations it was not necessary to pay attention to the shape of the boundary condition. Lets now suppose that the fluid flows in an area with a hydrophobic surface. Further, it is assumed that in a certain sub-region the shear stress vector $\sigma_{A}$ exceeds a critical size, at which the liquid slips.

Then the following inequality is obtained:

$\left|\sigma_{A}\right|>\left|\sigma_{K}\right|$.

On the boundary, we assume the validity of the following boundary condition 1 , when we change $\bar{v}=0$ for hydrophilic surface and $\kappa$ represents the adhesion coefficient.

$\boldsymbol{\sigma}_{A}-(\sigma \times m) \times \boldsymbol{m}=\kappa \boldsymbol{v}_{\Gamma}$.

Because it holds:

$\sigma_{i}=\widehat{\Pi}_{i j} m_{j}$,

The expression (4.2) can be written in a form: 
$\widehat{\Pi}_{j k} m_{k} m_{j} m_{i}-\widehat{\Pi}_{i j} m_{j}=\kappa v_{\Gamma i}$

where $\widehat{\Pi}_{j k}=2 \eta_{T} \hat{v}_{i j}-\frac{2}{3} \rho k \delta_{i j}$.

We note that for the numerical implementation of this boundary condition it is necessary to extend the model of turbulence with an additional boundary condition for $k$. Since $k$ is important for the pressure function, we propose the boundary condition for $k$ in the form:

$k=\xi \rho \hat{v}_{\Gamma i} \hat{v}_{\Gamma i}$.

The value of $\xi$ will depend on the surface quality and it must be obtained from an experiment.

\section{Experiment}

Verification of the effect of hydrophobic surface on hydraulic losses was performed on a tube of circular cross section with a diameter of $42.6 \mathrm{~mm}$ and a length of $6 \mathrm{~m}$ with water as as a viscous liquid representative. The differential pressure $\Delta p$ was measured by pressure sensors and the value of the specific energy was evaluated as:

$Y_{z}=\frac{\Delta p}{\rho}$,

For 4 types of surfaces - one hydrophilic - stainless steel $\theta=80^{\circ} \pm 3^{\circ}$

and three hydrophobic surfaces:

Steel UED - treated by the product Ultra Ever Dry developed by UltraTech international, Inc.: $\theta=$ $158^{\circ} \pm 1,5^{\circ}$

Ceramics: $\theta=95^{\circ} \pm 3^{\circ}$

Ceramics + UED: $\theta=157^{\circ} \pm 3^{\circ}$

The results are shown in the following Figures 5.1, $5.2,5.3$.

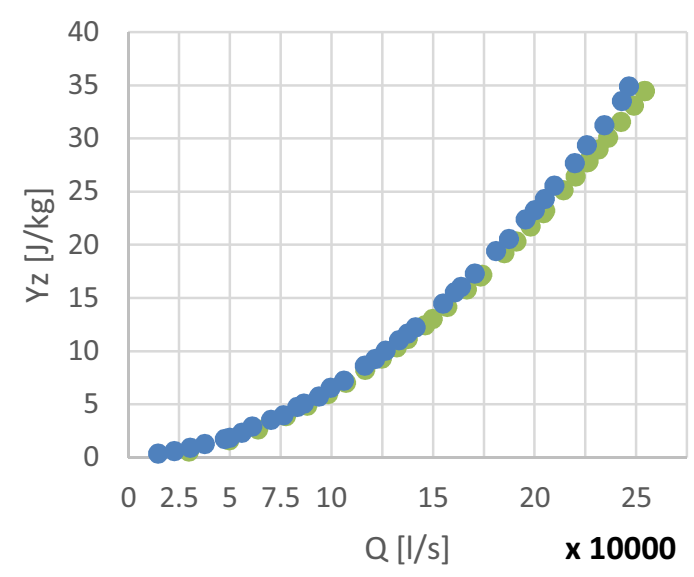

Fig. 5.1 Comparison of experimental results of the dissipation energy for two types of surfaces - green stailess steel, blue treated with UED

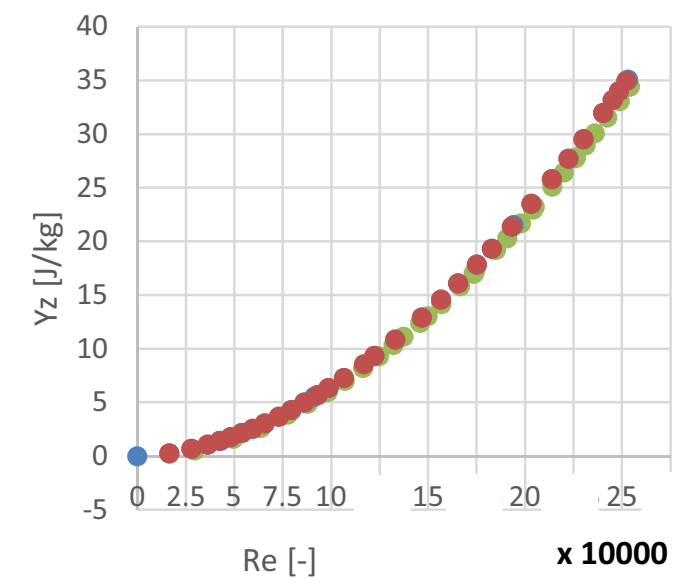

Fig. 5.2 Comparison of experimental results of the dissipation energy for three types of surfaces - green stailess steel, blue treated with UED, red ceramics

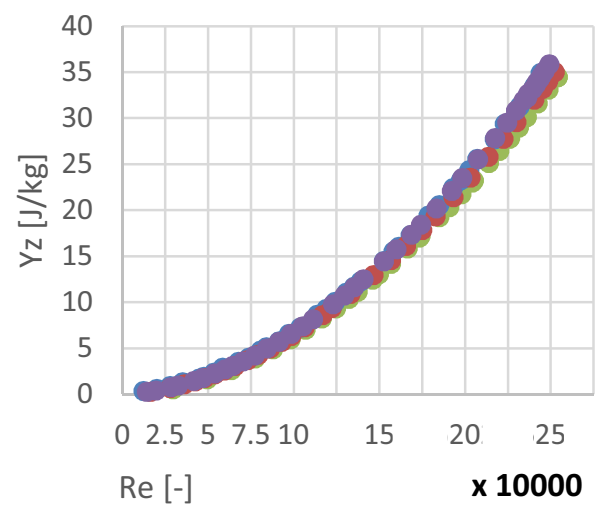

Fig. 5.3 Comparison of experimental results of the dissipation energy for four types of surfaces - green stailess steel (lowest in the graph), blue steel treated with UED, red ceramics, violet ceramics treated with UED (most upper in the qraph)

From Fig 5.1 it is clear that along a hydrophobic surface, the differential pressure increases with increasing flow rate and thus with increasing Reynolds number as compared to the hydrophilic surface. This result is very surprising since it is usually assumed that the differential pressure will be reduced when the slip of the liquid occurs close to the hydrophobic surface, compared with the hydrophilic surface.

However, considering the expression (2.19) for the pressure difference, it is clear that its value can be significantly affected by the size of turbulent viscosity. Turbulent viscosity on the wall is precisely the result of turbulent fluctuations that are caused by the liquid slip. That is the reason, that the pressure losses can be higher for higher Reynolds numbers. The value of the dissipation function may be in this case determined from relations (2.20) or (2.21). 


\section{Conclusion}

Within this work two very important relations were derived for the differential pressure values and dissipation function determination. Dissipation function characterizes the conversion of part of mechanical energy into heat:

$$
\begin{aligned}
\Delta \hat{p} & =\frac{\Gamma}{S}\left(\eta+\eta_{T}\right)|\operatorname{tg} \alpha| \\
2 \widehat{\mathcal{D}} & =\hat{P}=\left(\eta+\eta_{T}\right) \Gamma\left(v_{S}-v_{\Gamma}\right)|\operatorname{tg} \alpha| .
\end{aligned}
$$

From here it is clear that the values of $\Delta \hat{p}, \hat{P}$ stongly depend on the value of the angle $\alpha$ of the velocity profile on the wall of the tube, slip velocity and the value of the viscosity at the wall.

In the case of laminar flow $\eta_{T}=0$ and as a consequence of the liquid slip, angle $\alpha$ decreases, and thus also the value of $\Delta \hat{p}$ and $\hat{P}$ decreases in comparison with a hydrophilic surface.

Another situation occurs when the flow becomes turbulent. The angle $\alpha$, even though due to the slippage at the hydrophobic surface will decrease, the viscosity $\left(\eta+\eta_{T}\right)$ on the wall will rise up, so that $\Delta \hat{p}$ and $2 \widehat{\mathcal{D}}$ might increase.

However, situations may occur where in turbulent flow the value of $\Delta \hat{p}$ and $\hat{P}$ can drop down. This relates to both the shape of the area and the surface quality as well as the method of surface loading by shear stress; for example, a rotating disc near a hydrophobic surface.

In connection with the effects of a hydrophobic surface it is, however, important to realize that due to slippage of the liquid on the surface the vortex structures inside the area may significantly change. This explaines how the dissipation energy can differ in comparison with a hydrophilic surface.

Grant Agency of the Czech Republic, within the project GA101/16-18316S, is gratefully acknowledged for support of this work. Special thanks to Masaryk University, Faculty of Science, Dpt. of Physical Electronics and company ASIO, spol. s r.o..

\section{References}

1. Fialová S., Pochylý F., Identification and Experimental verification of the adhesive coefficient of hydrophobic materials, Wasserwirtschaft Extra, 1/2015, ISSN 0043 0978, pp. 125-129 (2015)

2. Jašíková, D. a Kotek, M., The Estimation of Dynamic Contact Angle of Ultra-hydrophobic Surfaces Using Inclined Surface and Impinging
Droplet Methods. EPJ Web of Conferences. Vol. 67, n. 0. pp. 1 - 6. ISSN 2100-014X. (2014)

3. Pochylý F., Fialová S., Kotek M., Zavadil L., Habán V., Volkov A.V., Parygin A.G., Utilization of hydrophobic layers in the design of hydraulic machines, EkopumpRus'2015 - sbornik dokladov, ISBN 978-5-9903138-5-9, printed, pp 77-83 (2015)

4. Versteeg H.K., Malalasekera W., An introduction to computational Fluid Mechanics. Longman, ISBN 0-582-21884-5, 257pages, (1995)

5. De Groot S.R., Mazur P.: Nonequilibrium thermodynamics, North-Holland Publishing, ISBN 0486647412, (1962)

6. Watanabe K., Yanuar,H. Udagawa, Drag reduction of Newtonian fluid in a circular pipe with a highly water-repellent wall, J. Fluid Mech. vol. 381, pp 225-238, (1999)

7. C. L. M. H. Navier, Mem Acad. Sci. Inst. France, vol. 1, pp. 414-416, (1823).

8. Olga I. Vinogradova, Slippage of water over hydrophobic surfaces, Int. J. Mineral. Process. 56, pp 31-60, (1999)

9. J.Ou, B.Perot, J.P.Rothstein, Laminar drag reduction in microchannels using ultrahydrophobic surfaces, Phys. Fluids 16, 4635-4643, (2004)

10. T.Min and J.Kim: Effects of hydrophobic surface on stability and transition. Physics of Fluids 17, 108106 (2005)

11. K.Kamrin, M.Z.Bazant,H.A.Stone, Effective slip boundary conditions for arbitrary periodic surfaces: the surface mobility tensor, J. Fluid Mech. vol. 658, pp 409-437, (2010)

12. B.R.K.Gruncelli, N.D.Sandham, G.McHale, Simulations of laminar flow past a superhydrophobic sphere with drag reduction and separation delay, Phys. Fluids 25, 043601, (2013)

13. P.Six, K.Kamrin, Some exact properties of the effective slipover surfaces with hydrophobic patterning, Phys. Fluids 25, 021703, (2013)

14. Daniello RJ, Waterhouse NE and Rothstein JP, Drag reduction in turbulent flows over superhydrophobic surfaces. Phys Fluids 21: 085103, (2009) 